\title{
Aspects épidémiocliniques des blocs auriculo-ventriculaires vus aux services de Cardiologie et USIC du CHU Befelatanana d'Antananarivo (Epidemioclinical Aspects of Atrioventricular Blocks Seen In the Services of Cardiology and USIC, CHU Befelatanana of Antananarivo)
}

\section{Ramiandrisoa Lahatriniavo Ritchy ${ }^{\star, \dagger}, 1$, Rakoto Sedson Rado Olivier ${ }^{2}$, Miandrisoa Rija Mikhaël ${ }^{3}$, Rakotoarimanana Solofonirina ${ }^{4}$, Rabearivony Nirina ${ }^{5}$}

\footnotetext{
${ }^{1}$ Service de Cardiologie, CHU Befelatanana, Antananarivo, Madagascar

${ }^{2}$ Service de Cardiologie, CHU Morafeno, Toamasina, Madagascar

${ }^{3}$ Service des Maladies Cardiovasculaires, Centre Hospitalier de Soavinandriana, Antananarivo, Madagascar

${ }_{4}^{4}$ Service des Soins Intensifs Cardiologiques, CHU Befelatanana, Antananarivo, Madagascar

${ }^{5}$ Service de Cardiologie, CHU Befelatanana, Antananarivo, Madagascar
}

(DDOI: https://doi.org/10.15520/jcmro.v2i10.208

Accepted 30-09-2019; Received 21-09-2019; Publish Online 01-10-2019

Reviewed By: Dr. JESTONI MANIAGO Department: Reviewer/CMRO

\section{ABSTRACT}

Aims: To establish the epidemiological and clinical aspects of atrioventricular block (AVB).

Patients and method: We have realised a descriptive retrospective study during 32 months from 1 January 2013 to 31 August 2015.

Results: The overall prevalence of AVB was $2.43 \%$. The average age of our patients was 61.38 years old with extremes of 17 and 89 . Men and the elder were the most affected. Hypertension was the most frequent cardiovascular risk factors. The circumstance of discovery was dominated by dyspnea. Bradycardia only affected $35 \%$ of patients. Signs of heart failure were present in $57 \%$ of cases. The majority of patients had a secondary AVB due to a hypertensive heart disease.

Conclusion: Atrioventricular block is a reality in cardiology practice in Madagascar. Taking care of hypertension could reduce its prevalence. Indeed, prevention should be intensified due to the cost of the pacemaker, still expensive for the majority of Malagasy people.

(Résumé :

But: Établir les aspects épidémiocliniques des Blocs Auriculo-Ventriculaires (BAV). Méthode: Nous avons effectué une étude rétrospective descriptive portant sur une période de 32 mois allant du $1^{\text {er }}$ Janvier 2013 au 31 Août 2015.

Résultats : La prévalence globale des BAV était de 2,43\%. L'âge moyen de nos patients était de 61,38 ans avec des extrêmes de 17 et 89 ans. Les hommes et les sujets âgés étaient les plus touchés. L'hypertension artérielle (HTA) constituait le facteur de risque cardio-vasculaire le plus fréquent. La circonstance de découverte était dominée par la dyspnée. La bradycardie n'avait concerné que $35 \%$ des patients. Les signes d'insuffisance cardiaque étaient présents dans $57 \%$ des cas. La majorité des patients avaient un BAV secondaire à une cardiopathie sous-jacente.

Conclusion: Le bloc auriculo-ventriculaire constitue une réalité dans la pratique médicale cardiologique à Madagascar. La prise en charge correcte de l'HTA pourrait réduire sa prévalence. En effet, la prévention devrait être intensifiée vue le coût du pacemaker, encore exorbitant pour la majorité de la population Malagasy.)

Key words: Bloc auriculo-ventriculaire-épidémiologie-Madagascar 


\section{Epidemioclinical Aspects of Atrioventricular Blocks Seen In the Services of Cardiology and USIC, CHU Befelatanana of Antananarivo

\section{INTRODUCTION:}

Le BAV désigne l'existence d'une perturbation (ralentissement ou interruption) de la transmission de l'influx électrique des oreillettes aux ventricules, à travers le nœud auriculo-ventriculaire, le faisceau de His ou simultanément dans les deux branches de ce faisceau [1] C'est une pathologie certes, peu fréquente mais qui s'observe bien partout dans le monde [2]. Selon l'OMS, l'aspect épidémiologique des BAV n'a pas encore été établi à l'échelle mondiale mais d'après une comparaison faite sur la population caucasienne et américaine, la prévalence était plus élevée dans la population américaine : 7,6\% contre $6,2 \%$ dans la population caucasienne [2]. En Afrique, des références retrouvaient une faible prévalence hospitalière : 1,6\% au Congo, $4,9 \%$ à Dakar [3, 4]. Pour Madagascar, aucune donnée récente n'est disponible sur cette maladie. Ainsi, nous proposons cette étude afin d'établir le profil épidémioclinique des BAV.

\section{MÉTHODE:}

L'étude s'est déroulée dans les services de cardiologie et de soins intensifs cardiologiques du CHU Befelatanana d'Antananarivo. Il s'agit d'une étude rétrospective descriptive s'étendant sur 32 mois (du mois janvier 2013 au mois d'Août 2015). Nous avons inclus les dossiers des patients dont l'ECG montrait un BAV (48 dossiers) et ont été exclus les dossiers jugés incomplets (8 dossiers).

Les éléments démographiques et cliniques étudiés étaient : le genre, l'âge, les facteurs de risque cardio-vasculaire, les habitudes médicamenteuses et/ou toxiques, la circonstance de découverte, la fréquence cardiaque (bradycardie si $<50$ et tachycardie si $>100$ par minute) et l'existence ou non de signes d'insuffisance cardiaque.

Les éléments paracliniques considérés étaient : l'ionogramme sanguin, la créatininémie, l'ECG de surface 12 dérivations qui a permis de poser le diagnostic des troubles de conduction et des arythmies associées, la fraction d'éjection du ventricule gauche échographique par la méthode de Teicholz et/ou Simpson Biplan : réduite si < $40 \%$, préservé si $50 \%$ et intermédiaire si entre 40 et $49 \%$.

Sur le plan étiologique, nous avons posé le diagnostic de BAV secondaires devant l'association à une cardiopathie sous-jacente : cardiopathie hypertensive, cardiopathie ischémique chronique, infarctus du myocarde (IDM) aigu, cardiopathie valvulaire, éthylique, du péri-partum ou cardiopathie dilatée de cause non retrouvée. Ensuite, le diagnostic de BAV iatrogènes étaient retenus devant une preuve d'imputabilité aux médicaments pouvant entrainer un trouble de conduction. Enfin, les BAV étaient classés idiopathiques lorsqu'aucune cause n'a été retrouvée.

\section{RÉSULTATS :}

Aspects épidémiologiques et cliniques :

* Corresponding author.

$\dagger$ Email: ritchyram@yahoo.com
Durant la période d'étude, le service avait pris en hospitalisation 1971 patients pour diverses affections. Parmi les patients hospitalisés, nous avons répertorié 48 cas de BAV soit une prévalence hospitalière de $2,43 \%$. Pour notre étude, 40 cas ont été retenus. L'âge moyen de nos patients était de 61,38 ans avec des extrêmes de 17 et 89 ans. Le genre masculin était concerné dans $57,5 \%$ des cas avec un sexe ratio à 1,35. L'HTA a été le principal facteur de risque cardiovasculaire, retrouvé chez $70 \%$ des patients Table 1 (tableau I). L'éthylisme concernait $40 \%$ des patients Table 1 (tableau I). La circonstance de découverte était dominée par la dyspnée Table 1 (tableau I). La majorité des patients ne présentait pas de bradycardie (65\%).Les signes d'insuffisance cardiaque étaient présents dans $57 \%$ des cas.

Données paracliniques:

Biologiquement, une insuffisance rénale était retrouvée chez 14 patients, soit $35 \%$ des cas. A l'échocardiographie, la FEVG étaient préservée dans 52,5\%, intermédiaire dans $7,5 \%$ et réduite dans $40 \%$ des cas. A l'ECG, le BAV type 2 et le BAV type 3 ne concernaient que le quart de notre population d'étude Table 1 (tableau I). Comme troubles du rythme associés, le BAV était apparu trois fois sur une fibrillation auriculaire. Il était associé à des troubles du rythme ventriculaire 7 fois dont 4 fois à des extrasystoles ventriculaires et 2 fois à des tachycardies ventriculaires nonsoutenues Table 1 (tableau I).

Données étiologiques :

Les BAV secondaires à une cardiopathie sous-jacente était les plus fréquentes dans $85 \%$ des cas. La cardiopathie hypertensive était au premier plan, retrouvée dans $40 \%$ des cas, puis la cardiopathie ischémique, qui avait concernée $22,5 \%$ de notre population d'étude. Les BAV iatrogènes ne concernait que $12,5 \%$ des patients et seulement $2,5 \%$ pour les BAV idiopathiques Table 2 (tableau II).

\section{DISCUSSION:}

Durant notre période d'étude, nous avons retenu 40 cas de BAV de tout type sur les 1971 malades hospitalisés dans le service, ce qui correspond à une prévalence globale de $2,03 \%$. Au Congo dans une série de 33 cas en 1992, la prévalence était de 1,6\%, comparable à notre étude [3]. Par contre en Europe et en Amérique, d'après une étude comparative faite en 2004 par Charles B et Upshaw J sur 1201 européens et 922 afro-américains, la prévalence était largement plus élevée respectivement de $7 \%$ et de 6,9\% [2]. Cette différence de prévalence s'expliquerait par la faible disponibilité de l'appareil ECG en Afrique, pouvant être responsable d'un sous-diagnostic.

Les facteurs de risque cardiovasculaire ont été dominés par l'HTA dans notre série (70\% des cas). Des références africaines rapportaient que les patients hypertendus étaient de 54,68 \% au Sénégal et 42,1 \% au Burkina Faso [3, 5]. Ceci reflète la place importante de l'HTA parmi les facteurs de risque cardiovasculaire. Le diabète n'a été observé que dans $7,5 \%$ de nos cas, dans $5,3 \%$ des cas dans la série de Sanou Z et 3,03\% dans celle de Nkoua J [3, 5] [3, 5]. 
Table 1. Répartition des patients selon les données de la clinique, de l'échographie Doppler cardiaque et le résultat de l'ECG d'effort.

\begin{tabular}{|c|c|c|}
\hline & $\begin{array}{l}\text { Ef- } \\
\text { fec- } \\
\text { tif }\end{array}$ & $\begin{array}{l}\text { Pour- } \\
\text { centage }\end{array}$ \\
\hline \multicolumn{3}{|l|}{ Circonstances de découverte } \\
\hline Syncope & 5 & 12,5 \\
\hline Vertige & 4 & 10 \\
\hline Douleur thoracique & 9 & 22,5 \\
\hline Dyspnée & 26 & 65 \\
\hline Palpitations & 4 & 10 \\
\hline (Edèmes de membres inférieurs & 12 & 30 \\
\hline \multicolumn{3}{|c|}{ Facteurs de risque et antécédents cardiovasculaires } \\
\hline HTA & 28 & 10 \\
\hline Diabète & 3 & 7,5 \\
\hline Dyslipidémie & 1 & 2,5 \\
\hline Tabagisme & 27 & 67,5 \\
\hline Obésité & 3 & 7,5 \\
\hline Sédentarité & 2 & 5 \\
\hline Hérédité cardiovasculaire & 19 & 47,5 \\
\hline \multicolumn{3}{|l|}{ Habitudes médicamenteux et toxiques } \\
\hline Cordarone & 5 & 12,5 \\
\hline Bêtabloquant & 5 & 12,5 \\
\hline Décoction & 2 & 5 \\
\hline Éthylisme & 16 & 40 \\
\hline Café & 6 & 15 \\
\hline \multicolumn{3}{|l|}{ Anomalies cliniques } \\
\hline $\begin{array}{l}\text { Bradycardie }(<50 \text { battements par } \\
\text { minute) }\end{array}$ & 14 & 35 \\
\hline Insuffisance cardiaque & 23 & 57,5 \\
\hline \multicolumn{3}{|l|}{ Anomalies biologiques } \\
\hline Insuffisance rénale & 14 & 35 \\
\hline Hyponatrémie & 8 & 20 \\
\hline Hypokaliémie & & 17,5 \\
\hline Hyperkaliémie & 7 & 5 \\
\hline Hypocalcémie & 2 & 2,5 \\
\hline Élévation de la troponinémie & 1 & 12,5 \\
\hline Anomalies électriques autre que le BAV & 5 & 5 \\
\hline Extrasystoles supraventriculaires & $\begin{array}{l}2 \\
3\end{array}$ & 7,5 \\
\hline Fibrillation auriculaire & $\begin{array}{l}3 \\
4\end{array}$ & 10 \\
\hline $\begin{array}{l}\text { Extrasystoles ventriculaires } \\
\text { Tachycardies ventriculaires non soutenues }\end{array}$ & $\begin{array}{l}4 \\
2\end{array}$ & 5 \\
\hline \multirow{6}{*}{$\begin{array}{l}\text { BBG complet } \\
\text { Anomalies échocardiographiques } \\
\text { Altération de la FEVG } \\
\text { FEVG intermédiaire } \\
\text { HVG } \\
\text { Trouble de la cinétique globale et } \\
\text { segmentaire }\end{array}$} & 14 & 35 \\
\hline & & \\
\hline & 19 & 47,5 \\
\hline & 7 & 17,5 \\
\hline & 12 & 30 \\
\hline & 19 & 47,5 \\
\hline
\end{tabular}

BAV : bloc auriculo-ventriculaire; BBG : bloc de branche gauche ; FEVG : fraction d'éjection ventriculaire gauche ; HVG : hypertrophie ventriculaire gauche

Dans notre étude, la circonstance de découverte était dominée par la dyspnée (65\%) suivie de loin par la syncope, notée seulement chez 6 patients (15\%). La syncope restait la manifestation la plus fréquemment rencontrée dans toutes les séries africaines avec des prévalences comprises entre 60 et $88,6 \%[3,6,7]$. Cette rareté de la syncope dans notre étude serait liée au fait que la majorité des malades ne présentaient pas de bradycardie. Quant à la dyspnée, elle était le maitre symptôme dans notre étude $(65 \%)$ contre $17 \%$ pour Ihenacho et $28 \%$ pour Sanou Z [8, 9] ; car les
Table 2. Répartition selon le type et l'étiologie des BAV

\begin{tabular}{lll}
\hline & $\begin{array}{l}\text { Ef- } \\
\text { fec- } \\
\text { tif }\end{array}$ & $\begin{array}{l}\text { Pour- } \\
\text { cent- } \\
\text { age }\end{array}$ \\
Type & 29 & 72,5 \\
BAV du premier degré & 2 & 5 \\
BAV du second degré & 9 & 22,5 \\
BAV complet & & \\
Étiologie & 5 & 12,5 \\
BAV iatrogène & 34 & 85 \\
BAV secondaire à une cardiopathie & & \\
sous-jacente & 1 & 2,5 \\
BAV idiopathique & & \\
Cardiopathies sous-jacentes rencontrées & 16 & 40 \\
Cardiopathie hypertensive & 9 & 22,5 \\
Cardiopathie ischémique & 1 & 5 \\
Cardiopathie hypertensive et ischémique & 4 & 10 \\
Cardiopathie éthylique & 2 & 5 \\
Cardiopathie du péripartum & 2 & 5 \\
Cardiopathie valvulaire & & \\
\hline
\end{tabular}

BAV : bloc auriculo-ventriculaire

BAV survenaient sur une cardiopathie décompensée dans la plupart des cas de notre série.

Sur le plan biologique, les signes retrouvés étaient surtout en rapport avec la cause du BAV ou avec les comorbidités. Cinq malades avaient présenté une élévation de la troponine I en phase aiguë d'un infarctus du myocarde responsable du BAV [8]. Chez un patient, nous avions rencontré une hypocalcémie sévère, pouvant être un facteur favorisant la survenue du trouble de la conduction [1]. Des troubles ioniques étaient aussi retrouvées telles qu'une hyponatrémie dans $20 \%$, une hypokaliémie dans $17,5 \%$ et une hyperkaliémie dans $5 \%$ des cas. Une insuffisance rénale était présente dans $35 \%$ des cas. Sanou Z et Alassane M ne retrouvaient dans leurs études qu'un cas d'élévation de la troponine I [4, 5]. L'hyponatrémie était moins fréquente dans notre étude que dans celle d'Alassane M (29,58 $\%$, mais plus fréquent que dans celle de Sanou Z (12 $\%)[4,5]$. Ces 2 auteurs retrouvaient des pourcentages plus bas d'hypokaliémie ( $6 \%$ et $12 \%)$ et d'hyperkaliémie $(1 \%$ et $3 \%)[4,5]$. Il en est de même pour l'insuffisance rénale qui était moins fréquente $(11 \%$ et $10 \%)$ par rapport à notre étude [4, 5].

Sur le plan électrique, l'ECG de surface a permis de relever $22,5 \%$ de BAV complet et $77,5 \%$ pour les autres types. Sanou $\mathrm{Z}$ et Alassane $\mathrm{M}$ ont retrouvé respectivement $78,9 \%$ et $57,6 \%$ de BAV complet $[4,5]$. Dans notre série, le BAV était associé à une fibrillation auriculaire chez 2 patients (5\%), et à des ESV chez 4 patients (10\%). Ces ESV devraient requérir une attention particulière, surtout lorsqu'elles surviennent sous forme de courtes salves ou d'un bigéminisme à couplage long et fixe [7]. Dans de telles conditions, elles seraient souvent prémonitoires d'accès typiques de torsade de pointe [7]. Certains auteurs ont aussi relevé l'association de troubles du rythme au BAV que ce soit supra-ventriculaire ou ventriculaire, corrélée avec un pronostic plus péjoratif $[2,6]$. 


\section{Epidemioclinical Aspects of Atrioventricular Blocks Seen In the Services of Cardiology and USIC, CHU Befelatanana of Antananarivo

Sur le plan étiologique, les BAV idiopathiques représentaient 2,5\% de notre population d'étude. En l'absence d'une cause secondaire évidente chez le sujet âgé, il était supposé d'origine dégénérative. L'étiologie dégénérative restait la plus fréquente avec $82 \%$ d'après Alassane $\mathrm{M}$ et $63 \%$ des cas d'après Sanou Z $[4,5]$. Elle serait due à une dégénérescence fibreuse des branches du faisceau de His (maladie de Lenègre), parfois à des lésions sclérocalcaires du tronc et de la bifurcation de ce faisceau (maladie de Lev). Ensuite, les BAV secondaires étaient retrouvés dans $85 \%$ des cas. Dans $42,5 \%$ des cas, le BAV survenait sur une cardiopathie d'origine hypertensive. Dans $25 \%$ des cas, une cause ischémique a été évoquée dont 5 cas d'infarctus aigu du myocarde, 3 cas d'angor instable et 2 cas de cardiopathie chronique d'origine ischémique. Les BAV secondaires étaient de 31,5\% pour Sanou Z et 6,1 \% pour Alassane M $[4,5]$. L'infarctus du myocarde était décrit avec des prévalences variables de 4,68\% dans la série d'Alassane à Dakar et 2,6\% dans la série de Sanou au Burkina Faso [4, 5]. Selon la littérature, l'infarctus inférieur s'accompagnerait d'un bloc suprahissien de bon pronostic; régressif, ne nécessitant pas en règle d'entraînement électrosystolique. Celui de l'infarctus antérieur serait de pronostic plus sévère et correspondrait à des lésions situées sur le faisceau de His nécessitant un entraînement transitoire voire définitif [8]. La cardiopathie hypertensive était moins fréquente dans l'étude de Sanou Z et al (13,5\%) que dans la nôtre, témoignant un contrôle insuffisant de l'HTA à Madagascar pour le moment [5]. Une cardiopathie éthylique compliquée de BAV a été retrouvée dans $5 \%$ dans notre série contre 2,6\% pour Sanou Z [5]]. Pour la cardiopathie du péripartum, nous avons trouvé 2 cas alors que les autres auteurs ne l'avaient pas décrite $[4,5]$. Enfin, 5 cas de BAV $(12,5 \%)$ ont été étiquetés d'origine iatrogène dont 2 cas dus aux bêtabloquants, 2 autres dus à l'amiodarone et 1 cas lié à une intoxication par la quinine. Alassane $M$ avait retrouvé 2 cas de BAV iatrogène, l'un dû au bêtabloquant et l'autre à la digitalique. Pour Sanou Z, les 2 cas qu'il avait retrouvé étaient tous liés au bêtabloquant [4, 5]. Selon la littérature, la conduction nodale serait déprimée par les digitaliques, les bêtabloquants, l'amiodarone, les inhibiteurs calciques et la quinine $[9,10]$. La conduction infra nodale serait déprimée par la plupart des anti- arythmiques de classe I et les imipraminiques [[9, 10]. Le BAV n'apparaîtrait qu'en cas d'intoxication ou lorsqu'il existerait un trouble conductif préalable (contre-indiquant leur emploi).

\section{CONCLUSION:}

Le bloc auriculo-ventriculaire constitue une réalité dans la pratique médicale cardiologique à Madagascar.

La majorité des patients étaient hypertendus et présentaient un BAV secondaire dont la cardiopathie hypertensive était la première cause. La prise en charge correcte de l'HTA pourrait ainsi réduire la prévalence de cette pathologie. En effet, la prévention devrait être intensifiée vue le coût de la pose de pace-maker, encore exorbitant pour la majorité de la population Malagasy.

\section{REFERENCES}

1. Djane P, Deharo JC. Blocs auriculo-ventriculaires. EMC Cardiologie Angéiologie. 1999;9(2):18-22 [Article 11-032-A-10].

2. Upshaw JR, Bernou C. Comparison of the Prevalence of Atrioventricular Block in African-American and in Caucasian Patients: An Electrocardiographic Study. J Natl Med Assoc. 2004 June; 96:756-60.

3. Nkoua JL, Bouramoué C. Les indications de la stimulation cardiaque définitive à Brazzaville: à propos de 33 cas. Cardiol Trop. 1992;18,70:53-7.

4. Alassane M, Kimbally-Kaki G. Troubles de la conduction auriculoventriculaire à Dakar, à propos de 64 cas. Cardiol Trop. 2005;87:20-69.

5. Sanou ZP. Blocs auriculo-ventriculaires au Burkina Faso: apports de la stimulation cardiaque [Thèse]. Médecine Humaine: Ouagadougou; 2001. 77p.

6. Ihenacho HC, Ebigbo PO, Ikeh VO. The african patients with cardiac pacemaker. Trop Cardiol. 1987;13,49:11-6.

7. Lévesque K, Maltret A, Hamidou M. Analyse des données de 82 blocs auriculo-ventriculaires inclus dans l'observatoire Français des lupus néonataux. Rev Méd Int. 2012 Juin;33:1-3.

8. Patanè S, Marte F. Paroxysmal Mobitz type-I atrioventricular block Luciani-Wenckebach conduction, acute myocardial infarction and severe three vessels coronary artery disease. Int $\mathrm{J}$ Cardiol. 2009;135:6-8.

9. Stavros G, Drakos, Maria I, Anastasiou N. Exacerbation of variant angina by metoprolol resulting in syncope due to transient atrioventricular block. Int J Cardiol. 2002;83:83-5.

10. Serinken M, Karcioglu O, Korkmaz A. Rarely seen cardiotoxicity of lithium overdose: Complete heart block. Int J Cardiol. 2008;98:52-63. 